\title{
Remembering the appearance of familiar objects: A study of monarchic memory
}

\author{
JOHN T. E. RICHARDSON \\ Brunel University, Uxbridge, England
}

\begin{abstract}
Two experiments were conducted to assess people's ability to remember the orientation of the monarch's profile on contemporary British coins. Experiment 1 confirmed that, when particular coins are drawn from memory, the likelihood of the Queen's head being drawn incorrectly as facing to the left was greater than chance. However, an identical bias appeared when other British subjects were simply asked to draw a female human profile. In Experiment 2, British subjects were asked to describe the orientation of the Queen's head on both coins and postage stamps. Most people were accurate in describing a 20-pence stamp but inaccurate in describing a 20-pence coin. The detailed findings indicated that many British people have a general schema for objects bearing the head of the monarch that is based primarily on their experience with postage stamps, but which is activated when they are asked to retrieve information about coins.
\end{abstract}

A number of different studies have shown that people's memory for the physical appearance of coins is very poor: they find it difficult to draw coins from memory, to recall their critical features, or to identify the correct representations of coins among plausible alternatives (Jones, 1990; Nickerson \& Adams, 1979; Rubin \& Kontis, 1983; Smyth, Morris, Levy, \& Ellis, 1987, pp. 209-210). One general tendency noted in several of these studies is for subjects to draw the obverse of coins with the head facing to the left when in reality it faces to the right. This finding was first noticed by Nickerson and Adams in the case of the American one-cent piece, and they attributed it to confusion with other American coins, on all of which at the time of their study the head faced to the left. In a similar manner, Rubin and Kontis posited some general schema or modal coin representation in which the head faced to the left. However, Jones noted a similar trend when British subjects attempted to draw contemporary British coins despite the fact that almost all U.K. coins in circulation showed the Queen's head facing to the right. He suggested that British people acquired a schema for objects bearing the monarch's head that was based on their experience with British postage stamps (on which the Queen's head faces to the left), but which was activated when they attempted to draw coins.

\section{EXPERIMENT 1}

One problem with all these studies is that people may simply have a general bias to draw human profiles as facing to the left, although this may depend on whether the drawer is left- or right-handed. Shanon (1979) investigated

Part of this paper was presented at the Fourth Conference of the European Society for Cognitive Psychology, Como, Italy, September 1990. The author is grateful for the comments and suggestions of Vicki Bruce, Gregory Jones, Peter Morris, and Benny Shanon. Requests for reprints should be sent to John T. E. Richardson, Department of Human Sciences, Brunel University, Uxbridge, Middlesex UB8 3PH, U.K. the incidence of such biases in a total of 160 American and Israeli subjects. In 80 subjects who consistently used their left hands to perform simple manual tasks, there was a tendency to draw right-facing rather than left-facing profiles in a proportion of $56 \%$ to $44 \%$. However, in the remaining 80 subjects, who consistently used their right hands to perform manual tasks, there was a tendency to draw leftfacing rather than right-facing profiles in a proportion of $81 \%$ to $19 \%$. This was not simply because they were imagining profiles on coins, since the same pattern of results was produced by Americans and by Israelis (whose coinage does not bear any human profiles). It is not strictly possible to extrapolate from these results to unselected samples of subjects because only $80 \%-85 \%$ of the general population are consistently left- or right-handed (see Newcombe \& Ratcliff, 1973). The following study was therefore carried out to compare biases in drawing coins and human profiles.

\section{Method \\ Subjects. Two successive cohorts of 110 and 149 students were tested during a first-year course in the social sciences. \\ Materials and Procedure. Both cohorts were asked to carry out a test of spatial ability, which was presented in a prepared booklet. The opening page requested certain items of biographical information, including "The hand you use or prefer to use for writing (left or right)" and "The hand you use or prefer to use for drawing (left or right)." The subjects then read the test instructions and proceeded to carry out the test itself for a period of $7 \mathrm{~min}$. This ensured that they were no longer aware that hand preference was a salient aspect of the study. At this point, both cohorts were asked to turn over their response booklets to reveal the blank final page. The first cohort was then asked to draw from memory the two sides of a 50-pence coin, although any newly arrived foreign students who were unfamiliar with British coinage were permitted merely to state that fact. The second cohort was asked to draw the face of a woman in profile; when they were finished, they were also asked to mention whether they had been thinking about a particular woman or some particular represen- tation of a woman and, if so, to identify the source of their drawing. \\ Results \\ Of the subjects in the first cohort, 17 stated that they were foreign students who were unfamiliar with British}


coinage, but all 93 of the other subjects did attempt to draw a 50-pence coin. Only 39 (or $42 \%$ ) correctly recalled its seven-sided shape, and the remainder produced drawings with five, six, eight, or nine sides. Seven subjects did not draw the Queen's head, and 9 drew it in full face; of the 77 subjects who drew the head in profile, only 23 (or $30 \%$ ) drew it correctly as facing to the right. These results are extremely similar to those obtained by Jones (1990), and this indicates that the present study was a successful replication of his own Experiment 3. Of the total sample of 110 subjects, 10 reported that they were lefthanded for drawing. Only 5 of the latter drew the Queen's head in profile, and all of these subjects drew it incorrectly as facing to the left. Out of the 72 right-handed subjects who drew the Queen's head in profile, 49 (or 68\%) drew it incorrectly as facing to the left.

Of the 149 subjects in the second cohort, 147 drew a woman's profile. As one might have anticipated from Shanon's (1979) results, 93 (or 63\%) of these profiles were facing to the left. Nineteen profiles were drawn by subjects who said they were left-handed for drawing, and 11 (or $58 \%$ ) of these were facing to the left; 128 profiles were produced by right-handed subjects, and 82 (or $64 \%$ ) of these were facing to the left. Of the total sample, 69 subjects gave no account of the source of their profiles, whereas 21 indicated that they had not been thinking about any particular source. Eight cited a generic type of woman (e.g., "a hag" or "a young woman with short hair"), 12 cited particular features of women (e.g., "softness" or "proud"), and another 12 cited abstract concepts (e.g., "cartoon" or "how to draw it"). Only 25 subjects cited a particular woman or a particular representation of a woman: 13 cited a personal acquaintance (e.g., "the girl next to me" or "'my mother"), 3 cited characters in fiction (e.g., "the Wizard of Oz"), and only 3 cited celebrities (e.g., "Kate Bush"); only 6 cited pictorial representations, of whom 4 cited the classical ambiguous "wife and motherin-law" figure and 2 cited illustrations from textbooks or advertisements. Not one subject cited the Queen or coins.

The results obtained in this experiment take the form of a three-way contingency table relating two fixed dichotomous factors (hand preference and task) to one random dichotomous factor (direction of profile). These data were analyzed by means of a partition of chi-square (see Winer, 1971, pp. 855-859). All obtained values of chi-square therefore had one degree of freedom. In the case of twoway comparisons, the obtained values were not subjected to Yates's correction for continuity, which is known to be inappropriate if one factor is random (see Richardson, 1990). There was a highly significant overall bias to draw left-facing profiles $\left(\chi^{2}=21.88, p<.001\right)$. However, this did not depend on the subjects' handedness $\left(\chi^{2}=0.01\right.$, $p>.9)$ or the subject's task $\left(\chi^{2}=1.06, p>.30\right)$, and there was no three-way interaction $\left(\chi^{2}=2.38, p>.10\right)$.

\section{Discussion}

The findings of this study are thus at variance with those of Shanon (1979), who found a bias to draw left-facing profiles among right-handed subjects but not among left-handed subjects. One possible explanation is that people who are consistently left-handed show essentially no bias when drawing human profiles, but that those who are left-handed for drawing but right-handed for at least some other activities are subject to roughly the same bias as those people who are consistently right-handed. The present investigation has of course supported the conclusion of Jones (1990) that drawings of coins by British people are systematically erroneous insofar as they tend to depict the monarch's head facing to the left rather than to the right. If this bias is interpreted to reflect the nature of the information about the physical appearance of coins that is represented in longterm memory, it is rather difficult to explain, since, unlike their counterparts in the United States, people in Britain nowadays tend only to encounter coins that bear a head facing to the right, which means that there is essentially no possibility of confusion or interference between memory representations of different coins. However, the results obtained here have demonstrated that the same pattern emerges when British people are simply asked to draw a female profile. In other words, their tendency to depict the head on a coin as facing in the wrong direction can plausibly be attributed to a general bias in drawing human profiles; consequently, in itself this tendency provides no evidence at all concerning the sorts of processes and representations that may be implicated in the storage of information about the physical appearance of familiar objects.

\section{EXPERIMENT 2}

A direct way to evaluate people's memory for the physical appearance of coins would be simply to ask them to say in which direction the head on a particular coin faced. The following experiment was therefore conducted to assess people's ability to recall the orientation of the monarch's head on contemporary British coins and also, given the specific theoretical assertion made by Jones (1990), on contemporary British postage stamps. All standard British postage stamps show the head of the Queen in profile and facing to the left, which means that stamps do represent a major potential source of confusion or interference with people's memory of the appearance of British coins. Nevertheless, postage stamps constitute a potentially interesting class of stimulus in their own right, because they possess a number of characteristics that have been suggested as factors leading to poor retention of the detailed physical appearance of coins, including relatively low levels of redundancy, meaningfulness, identifiability, and discriminativeness (Jones, 1990; Nickerson \& Adams, 1979).

\section{Method}

Subjects. This experiment involved 130 undergraduate students taking three different courses in the social sciences. Group A consisted of 70 first-year students, Group B consisted of 33 second-year students, and Group C consisted of 27 third- and fourth-year students.

Materials. Each subject was given an envelope containing two folded sheets of paper: one yellow, the other white. One piece of paper bore the question, "In which direction does the Queen's head face on a 20p coin?" The second piece of paper bore the question, "In which direction does the Queen's head face on a 20p stamp?" (At the time when this experiment was carried out, 20 pence was the basic cost of sending 
a letter by first class mail within the U.K. or to Europe.) For the subjects in Group A, both pieces of paper contained the two answers "(a) Towards the left" and "(b) Towards the right," printed one above the other; for the subjects in Group B and Group C, the two response alternatives were presented in the reverse order. Finally, each piece of paper bore the instruction, "Please tick ONE answer." Roughly half of the envelopes prepared for each group contained a yellow piece of paper bearing the question about the coin, whereas the remainder of the envelopes contained a yellow piece of paper bearing the question about the stamp. The envelopes were then combined together at random for later administration to the relevant subjects.

Procedure. The subjects were assessed as three groups during their normal academic activities. They were instructed to open their envelopes, to remove the yellow piece of paper, to answer the relevant question as quickly as possible without consulting their neighbors, and to reinsert the piece of paper in the envelope. They were then instructed to carry out the same process with the white piece of paper and, finally, to return the envelopes to the experimenter. No discussion or other conversation took place while the experiment was in process.

\section{Results}

The results obtained in this experiment take the form of a four-way contingency table relating two fixed dichotomous factors (order of tasks and order of response alternatives) and two random dichotomous factors (response to coin and response to stamp); this is shown as Table 1. The data were analyzed by means of a partition of chi-square, and all obtained values of chi-square had one degree of freedom. Once again, in the case of two-way comparisons, the obtained values were not subjected to Yates's correction for continuity, because this is known to be inappropriate when one or both factors are random (see Richardson, 1990).

When asked to indicate the direction in which the Queen's head was facing on a 20-pence coin, 79 (or $61 \%$ ) of the subjects responded that it faced to the left, whereas 51 (or $39 \%$ ) responded that it faced to the right; this pattern differed significantly from chance, but in the wrong direction $\left(\chi^{2}=6.03, p<.02\right)$. The relative frequency of "left" responses did not depend on the order of the tasks $\left(\chi^{2}=\right.$ $0.21, p>.9$ ): $63 \%$ of the subjects produced this response when asked about the coin first, and $59 \%$ did so when they were asked about the coin after having been asked about the stamp. There was, however, a clear effect of the order of the response alternatives $\left(\chi^{2}=7.23, p<.01\right): 71 \%$ responded "left" when this was the first of the two alternatives, but only $48 \%$ did so when it was the second. The net result was that, overall, $62 \%$ of the subjects chose the first response when answering the question about the coin, whereas only $38 \%$ chose the second response.

When asked to indicate the direction in which the Queen's head was facing on a 20-pence stamp, 97 (or $75 \%$ ) of the subjects responded that it faced to the left, whereas 33 (or $25 \%$ ) responded that it faced to the right; this pattern differed significantly from chance in the correct direction $\left(\chi^{2}=31.51, p<.001\right)$. The relative frequency of "left" responses did not depend on the order of the response alternatives $\left(\chi^{2}=0.51, p>.8\right): 77 \%$ responded "left" when this was the first of the two alternatives, and $72 \%$ did so when it was the second. However, there was an effect of the order of the tasks $\left(\chi^{2}=4.05, p<.05\right): 83 \%$ of the subjects responded "left" when asked about the stamp first, but only $67 \%$ did so when asked about the stamp after having been asked about the coin.

In addition, there was a clear association between the response given about the coin and that given about the stamp $\left(\chi^{2}=13.96, p<.001\right): 70 \%$ of those subjects who responded "left" in the case of the stamp also did so in the case of the coin, but only $33 \%$ of those who responded "right" in the case of the stamp responded "left" in the case of the coin; conversely, $86 \%$ of those subjects who responded "left" in the case of the coin also did so in the case of the stamp, but only $57 \%$ of those who responded "right" in the case of the coin responded "left" in the case of the stamp. Of the total sample of 130 subjects, 90 (or $69 \%$ ) gave the same response to both questions, whereas 40 (or $31 \%$ ) gave different responses, and only 29 (or $22 \%$ ) gave the correct combination of responses.

The three-way association between the order of the two tasks, the response given about the coin, and the response given about the stamp approached statistical significance $\left(\chi^{2}=2.81, p<.1\right)$. In the case of the question about the coin, the order of the tasks had no effect on the frequency of "left" responses, regardless of whether the subjects responded "left" $\left(\chi^{2}=2.34, p>.1\right)$ or "right" $\left(\chi^{2}=0.07, p>.7\right)$ to the question about the stamp. In the case of the question about the stamp, the order of the tasks had no effect on the frequency of "left" responses if the subjects responded "left" to the question about the coin $\left(\chi^{2}=0.56, p>.30\right)$. However, if the subjects responded "right" to the question about the coin, the order of the tasks did affect the responses given to the

Table 1

Contingency Table Relating Order of Tasks, Order of Response Alternatives, and Reported Orientation of the Queen's Head on a 20-pence Coin and a 20-pence Stamp

\begin{tabular}{lccrrrr}
\hline & & \multicolumn{2}{c}{ CL } & & \multicolumn{2}{c}{ CR } \\
\cline { 3 - 5 } \cline { 5 - 7 } Order of Tasks & Order of Responses & SL & SR & & SL & SR \\
\hline Coin-stamp & Left-right & 22 & 6 & 4 & 4 \\
Stamp-coin & Right-left & 13 & 1 & 6 & 11 \\
& Left-right & 19 & 3 & 9 & 3 \\
Totals & Right-left & 14 & 1 & 10 & 4 \\
& & 68 & 11 & 29 & 22 \\
\hline
\end{tabular}

Note-CL $=$ the head on a 20 -pence coin was said to be facing to the left; $C R=$ the head on a 20-pence coin was said to be facing to the right; $S L=$ the head on a 20 pence stamp was said to be facing to the left; $S R=$ the head on a 20-pence stamp was said to be facing to the right. 
question about the stamp $\left(\chi^{2}=5.68, p<.02\right): 73 \%$ of subjects responded "left"' to the question about the stamp if it was asked first, but only $40 \%$ did so if they had previously responded "right" to the question about the coin.

The three-way association between the order of the two response alternatives, the response given about the coin, and the response given about the stamp also approached statistical significance $\left(\chi^{2}=3.49, p<.1\right)$. In the case of the question about the stamp, the order of the two response alternatives had no effect on the frequency of "left" responses, regardless of whether the subjects responded "left" $\left(\chi^{2}=1.89, p>.1\right)$ or "right" $\left(\chi^{2}=0.89\right.$, $p>$.3) to the question about the coin. There was no overall tendency in either situation for the subjects systematically to choose the first response alternative rather than the second. In the case of the question about the coin, the order of the response alternatives had no effect on the frequency of "left" responses if the subjects responded "left" to the question about the stamp $\left(\chi^{2}=1.97, p>.1\right)$. However, if the subjects responded "right" to the question about the stamp, the order of the response alternatives did affect the responses that were given to the question about the coin $\left(\chi^{2}=7.34, p<.01\right): 56 \%$ responded "left" to the question about the coin if it was the first alternative, but only $12 \%$ responded "left" if this was the second alternative. No other terms in the partition of chi-square even approached statistical significance.

\section{Discussion}

This experiment has shown that three quarters of British students are able to report the direction in which the Queen's head faces on a 20-pence stamp. They seem to be less certain about the stamp when they have been previously asked about the direction in which the Queen's head faces on a 20-pence coin and were able to report correctly that the Queen's head faced to the right on the coin. Nevertheless, in general, their long-term memory for the gross physical appearance of postage stamps appears to be relatively good. A different pattern of results emerges when the same subjects are asked to describe the direction in which the Queen's head faces on a 20-pence coin. More than $60 \%$ give the incorrect answer, and their responses appear to be based on one of two strategies. First, they may refer to their memory representations of other familiar objects that depict the monarch's head-namely, postage stamps: $69 \%$ of the subjects tested gave the same response to the question about a 20-pence coin as they did to the question about a 20-pence stamp. Second, they may guess and simply choose whichever is the first of the response alternatives offered; indeed, of those subjects who responded incorrectly that the Queen's head faced to the right on a 20-pence stamp, $73 \%$ chose the first of the two alternatives rather than the second when asked to say in which direction the Queen's head faced on a 20pence coin.

\section{GENERAL DISCUSSION}

This account implies that in trying to recall the physical appearance of coins with which they are very familiar many people have no information about the orientation of the head, either specific or schematic. In turn, the present findings confirm that people may have no reliable memory even for salient physical characteristics of familiar everyday objects. Some authors have pointed out that people simply do not need to have detailed knowledge of the physical appearance of coins to use them correctly but do so merely on the basis of gross features such as their color and size (Morris, 1988; Nickerson \& Adams, 1979; Norman, 1988, pp. 58-59). Postage stamps, in contrast, are customarily attached to envelopes the right way up, and the orientation of the monarch's head is a primary guide to this end. It follows that people will have a much better basis for establishing the direction in which the Queen's head faces on postage stamps than they will on coins (V. Bruce, personal communication, April 16, 1990). It is, however, important to note that coins and stamps are broadly similar to each other with respect to a number of other characteristics that have been suggested as possible factors leading to the poor retention of the detailed physical appearance of coins, such as their redundancy, meaningfulness, identifiability, and discriminativeness (see Jones, 1990; Nickerson \& Adams, 1978). Such alternative explanations are ruled out by the relatively good retention of the physical appearance of postage stamps that was obtained in the present investigation.

Nevertheless, none of these explanations is able to handle the more fundamental problem that, in Britain, people's memory for the orientation of the head on coins is not simply poor but "systematically erroneous," even though current British coinage is almost entirely consistent in depicting the head of Queen Elizabeth II as facing to the right. This poin was made previously by Jones $(1990$, p. 181$)$ on the basis of studies in which people had been asked to draw coins from memory, but the results of Experiment 1 appear to have discredited such research as not providing any evidence at all concerning the representation of information about the physical appearance of familiar objects because of the problem of biases in drawing profiles. Despite this, a similar tendency to recall the Queen's head incorrectly as facing to the left was apparent in the present experiment when any general biases in drawing profiles had been wholly eliminated by means of a simple two-choice response paradigm. Jones speculated that British people tend to acquire a general schema for objects bearing the monarch's head that is based on their experience with postage stamps, but which is activated when they are asked to retrieve information about coins. The detailed findings of the present experiment offer direct confirmation of such a proposal.

\section{REFERENCES}

JoNes, G. V. (1990). Misremembering a common object: When left is not right. Memory \& Cognition, 18, 174-182.

MoRRIs, P. E. (1988). Expertise and everyday memory. In M. M. Gruneberg, P. E. Morris, \& R. N. Sykes (Eds.), Practical aspects of memory: Current research and issues: Vol. 1. Memory in everyday life (pp. 459-465). Chichester, U.K.: John Wiley.

NewCOMBE, F., \& RaTcLIF, G. (1973). Handedness, speech lateralization and ability. Neuropsychologia, 11, 399-407.

Nickerson, R. S., \& ADAMS, M. J. (1979). Long-term memory for a common object. Cognitive Psychology, 11, 287-307.

Norman, D. A. (1988). The psychology of everyday things. New York: Basic Books.

Richardson, J. T. E. (1990). Variants of chi-square for $2 \times 2$ contingency tables. British Journal of Mathematical \& Statistical Psychology, 43, 309-326.

Rubin, D. C., \& Kontis, T. C. (1983). A schema for common cents. Memory \& Cognition, 11, 335-341.

ShanON, B. (1979). Graphological patterns as a function of handedness and culture. Neuropsychologia, 17, 457-465.

Smyth, M. M., Morris, P. E., Levy, P., \& Ellis, A. W. (1987). Cognition in action. London: Erlbaum.

WINER, B. J. (1971). Statistical principles in experimental design (2nd ed.). New York: McGraw-Hill. 